\section{Entre exiliados y nativos: la integración de saberes de españoles y mexicanos para el desarrollo de la neurología en México, 1935-1950}

\section{Between exiled and native doctors: Spanish and Mexican knowledge integrating into the development of neurology in México, 1935-1950}

VICENCIO, Daniel. Entre exiliados y nativos: la integración de saberes de españoles y mexicanos para el desarrollo de la neurología en México, 1935-1950. História, Ciências, Saúde - Manguinhos, Rio de Janeiro, v.28, n.3, jul.-set. 2021, p.709-725.

\section{Resumen}

Entre 1935 y 1950 aconteció la introducción y desarrollo de la neurología en México. Esto se dio por medio de dos vías: el arribo de los neurocientíficos españoles a México tras su exilio provocado por la Guerra Civil; y la presencia de médicos mexicanos que salieron a especializarse en neurocirugía a EEUU. Se discuten algunas posiciones historiográficas que hablan de la importancia de los españoles exiliados en este acontecer, pero que no han expuesto el relevante papel de los nativos en el surgimiento de la neurología mexicana. Se afirma la existencia de un proceso de integración de ambas partes, donde los nativos buscaron satisfacer necesidades asistenciales mientras que los exiliados tuvieron que encontrar y crear espacios dónde insertarse.

Palabras clave: neurología; médicos mexicanos; españoles en exilio; historia de México; proceso de integración.

\section{Abstract}

Between 1935 and 1950 the neurology was presented and developed in Mexico. It happened by two ways: the arrival of Spanish neuroscience researchers in Mexico exiled due the Civil War; and the presence of Mexican doctors that had specialized in neurosurgery in the United States. The article discusses historiographic points of view that stress the importance of the Spanish exiled doctors, but neglect the important role of native doctors in the emergence of Mexican neurology. It states that there was an integration process by both parts, where Mexicans tried to satisfy care needs while the Spanish had to find or create working spaces to belong to.

Keywords: neurology; Mexican doctors; Spanish in exile; history of México; integration process. 
$F_{y}^{u}$ ue entre 1935 y 1950 que la neurología se introdujo a México a través de la neurocirugía y las neurociencias. Tales disciplinas fueron importadas de otros espacios que pasaron por una integración a lo local, ya que ellas se gestaron por medio de saberes que arribaron de diferentes partes, primordialmente España y EEUU, lo que influyó para que en su devenir histórico existieran debates, negociaciones, enfrentamientos y conformación de redes en torno a los procesos de construcción de conocimiento y de práctica médico-científica.

Aquí se hace referencia a la neurología como un término que contempla esa disciplina, la neurocirugía y las neurociencias, aunque sin negar que cada una haya tenido sus propias características y elementos identitarios; esto se hace con fines prácticos para exponer mejor las ideas del texto, y no se pretende afirmar que las segundas dependan o estén englobadas en la primera. Los practicantes de las tres fueron los que incorporaron al país los nuevos saberes clínicos y científicos sobre el sistema nervioso, y formaron parte de una misma comunidad de profesionistas. En ese sentido, se han identificado dos grupos de especialistas en esas áreas: unos fueron españoles, exiliados en México, y los otros mexicanos que se instruyeron en EEUU. Ellos por medio de su trabajo en conjunto y sus relaciones, a veces cooperativas y otras ríspidas, iniciaron una vinculación de conocimientos que a la larga fue la base para el surgimiento de la neurología en el país.

Ahora bien, al analizar la historiografía que hay al respecto, existen autores que han expuesto la importancia que tuvieron los exiliados españoles para el desarrollo de las neurociencias y la neurología en México. Afirman que tras la Guerra Civil española (1936-1939) algunos médicos que se dedicaron a la investigación neurocientífica y a la neurocirugía llegaron a México como exiliados y mencionan que sus aportes en estas ramas de la medicina científica fueron fundamentales para la neurología y para la conformación de una comunidad médica en su país de acogida. Tales autores han sugerido que gracias a esos españoles dichas disciplinas tuvieron un despunte en el país, debido a que trajeron un bagaje de conocimientos que pusieron en práctica en instituciones de investigación y hospitalarias mexicanas. Y comentan que una de las principales razones de eso es porque los exiliados fueron herederos del trabajo de Santiago Ramón y Cajal, quien fue un importante científico e hizo invaluables aportes para el estudio del sistema nervioso a finales del siglo XIX y principios del XX (Dosil Mancilla, 2010, 2009, 2007; Cruz-Sánchez et al., 2002; Fernández Guardiola, 1997).

Sin embargo, esa historiografía omite que los españoles no fueron los únicos que participaron en la introducción de tales disciplinas en el país. Resulta que poco tiempo después de su llegada, un grupo de médicos mexicanos comenzó a salir al extranjero, particularmente a EEUU, a especializarse en neurocirugía. A su regreso a México también desempeñaron un papel indispensable en la expansión de las neurociencias, la neurología y la neurocirugía.

Lo que ellos aprendieron en Estados Unidos fue relevante, ya que ese país tuvo un avance bastante significativo en el terreno de la neurocirugía y la investigación neurológica durante la primera mitad del siglo XX, de la mano de médicos como Harvey Cushing (1869-1939), Walter E. Dandy (1886-1946), Charles Elsberg (1871-1948) o Charles H. Frazier (1870-1936), quienes implementaron las primeras operaciones descompresivas subtemporales, la hemisterectomía o la neurocirugía cerebrovascular (Hodelín Tablada, 
2011; Sherman et al., 2006). Estos y otros más son conocidos como los iniciadores de la neurocirugía contemporánea; sus técnicas se expandieron a lo largo del mundo, y hacia los años 1930, esa disciplina se estableció como una especialidad con bases sólidas, científicas y terapéuticas. En ese sentido, EEUU tenía mucho que ofrecer en esa rama a los jóvenes estudiantes.

Entonces, hacia la década de 1940 algunos médicos mexicanos viajaron al vecino país del Norte para convertirse en neurocirujanos, con el objetivo de volver y poner en práctica en instituciones hospitalarias públicas todo lo aprendido; como en efecto ocurrió. No obstante, su papel en la neurología no ha sido analizado históricamente. En ese sentido, el aporte que aquí se plantea es mostrar que no solo fueron los españoles quienes participaron en el surgimiento y desarrollo de esas disciplinas en México, sino que hubo otra vía fundamental para su introducción, ya que los nativos que salieron a instruirse también llevaron a cabo un importante trabajo en la clínica, en la investigación y en la creación de espacios hospitalarios y de investigación para la expansión de su especialidad. Al final, unos y otros pudieron desarrollar su labor porque en México había condiciones para ello.

Así, el objetivo de este trabajo es observar la labor de los dos grupos de médicos, como actores independientes y como miembros de una misma comunidad, para mostrar que lo que ocurrió fue un "proceso de integración" de los conocimientos de ambos, que se vio afectado por motivaciones diversas e intereses prácticos en ocasiones distintos. Ese último argumento es relevante ya que no hubo una imposición de saberes de alguno de los grupos sobre el otro.

El concepto de integración resulta importante; aquí se define como el medio por el cual el conocimiento se vincula a una estructura definida o en conformación, donde se identifican saberes en común, lazos existentes y redes creadas a partir de colaboraciones; "de esa manera la integración viene a ser aquella operación por medio de la cual [se hacen] interdependientes y solidarios elementos que estaban disociados al inicio, propiciando que [el conocimiento] pueda funcionar de manera articulada" (Vélez Cardona, 2013, p.646). George Weisz está de acuerdo en que uno de los elementos para comprender la historia de las especialidades médicas, como es la neurología, es observar la forma en la que estas se integraron en las redes existentes en el campo de lo médico y en la sociedad en general. En ese sentido, el autor comenta que deben existir grupos de actores (profesionistas) que se vinculen para conformar asociaciones especializadas; ellos son los que transformarán a las instituciones y se convertirán, en algunos casos, en materia de interés para autoridades políticas. Weisz (2005, p.XI-XVIII) emplea el concepto "sistemas de especialidades", que se define como la integración de individuos que rigen, regulan, estandarizan y transforman los parámetros y las prácticas de la especialidad, y que colectivamente colaboran con sujetos e instituciones que constituyen su propio medio social (médicos, pacientes, hospitales, estado, universidades). Los "sistemas" negocian con las redes institucionales, y así es como se establecen las especialidades médicas.

En realidad aquí no se hablará de un "sistema de especialidad" neurológica en sí, ya que eso solo podría ser comprendido mediante el análisis del complejo tejido social de la época estudiada; pero lo que sí se hará es observar uno de los elementos que, a mi juicio, determina a las especialidades, que en este caso es el proceso de integración de conocimientos de los 
actores que condicionaron la eclosión de una comunidad médica; ello permitirá comprender cómo es que españoles y mexicanos se vincularon para producir el surgimiento y avance de la neurología en el país.

\section{Los neurocientíficos españoles}

En 1936 inició la Guerra Civil española cuando el Ejército dirigido por el general Francisco Franco se levantó en armas contra el gobierno republicano; como consecuencia de ello una gran cantidad de personas, entre ellos médicos y científicos, salieron de su país hacia 1939. El entonces presidente de México, Lázaro Cárdenas (1934-1940), rechazó el levantamiento del Ejército español y apoyó a la República en distintos rubros, pero quizá la ayuda más importante fue el acogimiento de miles de refugiados en territorio nacional (aproximadamente 20 mil) (Lida, 1997, p.57), de los cuales un gran número se dedicaba a una profesión médica (Guerra, 1996, p.11).

Algunos de esos médicos que llegaron a México se habían interesado por las neurociencias, la neurología y la neurocirugía desde que fueron estudiantes, y eso tuvo que ver con la presencia de Santiago Ramón y Cajal. Él fue un científico español especializado en histología y anatomía patológica. Gracias a sus descubrimientos, hechos a finales del siglo XIX sobre el sistema nervioso, pudo escribir su obra Histología del sistema nervioso del hombre y los vertebrados, con la que obtuvo el Premio Nobel de Fisiología y Medicina en 1906. Las investigaciones que le llevaron a recibirlo giraron en torno a los mecanismos que gobiernan la morfología y los procesos conectivos de las células nerviosas, con lo cual expuso una nueva teoría que afirmaba que el tejido cerebral está compuesto por células individuales. Eso lo pudo mostrar gracias a sus amplios conocimientos, por ejemplo sobre tinción, y a su competencia como microfotógrafo.

Sus descubrimientos fueron reconocidos por el gobierno, y en 1900 creó para él el Instituto de Investigaciones Biológicas, que en 1932 pasó a llamarse Instituto Cajal; este se convirtió no solo en un centro dedicado a la investigación neurocientífica, sino también en un símbolo del desarrollo científico español (aunque fue desmantelado tras la Guerra Civil). Con todo ello, gracias a Cajal, se creó la "escuela histológica española" y se sentaron las bases de la investigación neurológica en ese país, desarrolladas en el primer tercio del siglo XX (Rodríguez Quiroga, 2002, p.412; Baratas Díaz, 2007, p.336).

En opinión de Laín Entralgo (1978, p.58), Cajal encabezó una generación de sabios formada en el último tercio del XIX. Él difundió sus descubrimientos desde la década de 1890, dando conferencias y publicando en las que entonces eran las lenguas de la ciencia: inglés, francés y alemán; aunado a eso, a raíz de la obtención del Premio Nobel, la difusión de sus trabajos tuvo un mayor empuje y por ello hubo en el mundo "un notable interés por todas las concepciones de Cajal" (Nieto, 1990, p.19). Fue por eso que generó un gran impacto en otros científicos y tuvo diversos discípulos. Se puede decir que Cajal representaba ante todo la encarnación de un ethos científico.

Al lado de él, muchos médicos jóvenes españoles comenzaron a interesarse en la investigación neurocientífica, neurobiológica y neurofisiológica. Pero también ellos fueron a otros países europeos donde los estudios sobre el sistema nervioso tenían características 
propias, y eso permitió que el avance científico español, hablando de esas ramas de estudio, se nutriera de otros saberes. Esto fue posible gracias a las pensiones otorgadas por la Junta de Ampliación de Estudios (JAE).

La JAE (1907-1939) fue una institución que tuvo por misión convertirse en el motor científico y humanístico de España por medio, entre otras cosas, del otorgamiento de pensiones a estudiantes aventajados para que tuvieran una formación en el extranjero (Dosil Mancilla, 2007, p.308). El hecho de que Cajal fuera uno de los impulsores de la JAE y su primer presidente repercutió en que "la investigación en biología en general, y en neurobiología en particular, figurara entre las áreas más activas" (Baratas Díaz, 2007, p.335). Los españoles dedicados a las neurociencias, pensionados por la JAE, obtuvieron gran parte de su preparación en las mejores escuelas europeas de su tiempo y por eso fue que importaron a México todos esos saberes acumulados. Su preparación era excepcional.

Diversos especialistas en neurociencias, neurología y neurocirugía llegaron a México en el exilio. Algunos de ellos fueron: Gonzalo Rodríguez Lafora (1886-1971), Wenceslao López Albo (1889-1944), Sixto Obrador Alcalde (1912-1978) y Dionisio Nieto (1905-1987). Gonzalo Rodríguez Lafora fue alumno distinguido de Cajal y después estudió anatomía del sistema nervioso en Berlín y Munich, Alemania, junto a médicos de la talla de Emil Kraepelin (1856-1926) (quien hizo importantes aportes clínicos en psiquiatría y propuso las categorías de demencia precoz y psicosis maniaco depresiva) y Alois Alzheimer (1864-1915) (quien realizó diversos estudios en el campo de la neurología y describió la enfermedad que lleva su nombre). Llegó a México en 1938, donde se relacionó con los psiquiatras que trabajaban en el Manicomio General La Castañeda y fue nombrado miembro honorario de la Academia Nacional de Medicina. Fue uno de los fundadores del Laboratorio de Estudios Médicos y Biológicos (institución dedicada, entre otras cosas, a la investigación neurocientífica) y del Instituto Neuropsiquiátrico (institución privada que ofrecía clínica psiquiátrica); regresó a España en 1947 (Huertas, 2002).

Wenceslao López Albo llegó a México en 1939. Se inició en neurología con el médico español Nicolás Achúcarro y se especializó en neurocirugía en Berlín, Alemania, y París, Francia. En un principio se instaló en Nuevo León, donde trabajó como neurocirujano y fue profesor de neurología y psiquiatría en la facultad de medicina. En 1942 se trasladó a la Ciudad de México, donde trabajó en el Manicomio General; cofundó el Instituto Neuropsiquiátrico y trabajó en el Hospital Español. Murió por una infección paratífica, en 1944. Se interesó sobre todo en el estudio de la cisticercosis cerebral y el diagnóstico neuropsiquiátrico (Guerra, 2003, p.579).

Sixto Obrador Alcalde obtuvo una beca de la JAE para realizar estudios de neurocirugía en Inglaterra. Llegó a México en 1940 y se instaló en Nuevo León. Al poco tiempo viajó a la Ciudad de México, donde se desempeñó como neurocirujano en el Hospital Español, en el Instituto Neuropsiquiátrico y en el Manicomio, lugares donde trabajó con López Albo, quien fue uno de sus mentores. También se dedicó a la investigación experimental en neurología y trabajó en el Laboratorio de Estudios Médicos y Biológicos. Regresó a España en 1945 (Guerra, 2003, p.580; Giral, 1994, p.257).

Dionisio Nieto fue pensionado por la JAE en 1932 para hacer investigación neurológica en el Instituto Max Plank de Munich y clínica psiquiátrica en las Universidades de Berlín 
y Hamburgo, en Alemania. Fue investigador en el Instituto Cajal hasta que se exilió en México. Llegó en 1940 y fue contratado como jefe del Laboratorio del Manicomio General. Ahí Nieto comenzó una labor de investigación y reunió cerebros humanos para seguir con sus estudios sobre la patogenia de las enfermedades mentales. También, trabajó en el Laboratorio de Estudios Médicos y Biológicos. Se orientó a estudiar los fundamentos biológicos de la enfermedad mental y fue un decidido impulsor de la psiquiatría biológica y del uso de psicofármacos (Sacristán, 2007, p.330-339; Fernández Guardiola, 1997, p.4168). Permaneció en México hasta su muerte.

Esos médicos se describen aquí con más detalle porque se especializaron en neuropsiquiatría, neurociencias y/o neurocirugía; sin embargo, hubo otros que también realizaron estudios concernientes al sistema nervioso, como el histólogo Isaac Costero, el neurofisiólogo Ramón Álvarez-Buylla, los fisiólogos Jaime Pi Suñer y Rosendo Carrasco Formiguera o los oftalmólogos Manuel Márquez Rodríguez y Manuel Rivas Chérif.

Así, en opinión de Dosil Mancilla (2009, p.30), con la llegada de ellos, "los científicos mexicanos encontraron a menudo en la escuela de Cajal criterios para guiar metodológicamente sus investigaciones y su desempeño profesional". Tal postura la apoyan otros como Fernández Guardiola, Félix Cruz-Sánchez y Díaz. Este último, por ejemplo, menciona: "Muchos neurocientíficos mexicanos nos consideramos, en cierta medida, descendientes o, como mínimo, beneficiarios de la escuela de Santiago Ramón y Cajal" (Díaz, 2009, p.1). Dichos autores exponen la importancia del arribo a México de esos personajes y otros interesados por la investigación histopatológica y conocedores de la escuela de Cajal. Y esas afirmaciones tienen sustento, ya que es cierto que los exiliados realizaron en México investigación básica y clínica; crearon laboratorios y hospitales; participaron en sociedades científicas; y tuvieron discípulos que instruyeron con sus amplios saberes.

Sin embargo, no es posible afirmar que lo que se hizo en México con relación a la neurología partió solo de lo que los españoles trajeron. Existieron otros personajes, nativos de México, que también se especializaron en esas ramas de la medicina, específicamente en EEUU y que igualmente importaron conocimientos que no estaban expresamente ligados de la escuela cajaliana o por lo menos no eran herederos directos de ella. El caso de aquellos se verá más adelante. No obstante, lo que se quiere expresar por el momento es que lo que los españoles importaron no fue lo único que en ese entonces permeó el panorama neurocientífico; en ese sentido es que se habla de un "proceso de integración" de saberes, relaciones y prácticas, entre lo que aportaron los acogidos y lo que en México se desarrollaba. $\mathrm{Y}$ es que en realidad no todo lo que importaron los españoles fue recibido sin debates o negociaciones.

Lo dicho anteriormente puede observarse en situaciones específicas que los españoles vivieron en el país de acogida. Lafora, por ejemplo, tuvo conflictos con otros miembros de la medicina mexicana cuando hizo un estudio clínico del famoso asesino Gregorio Cárdenas, quien a inicios de la década de 1940 asesinó a cuatro mujeres y las enterró en el patio de su casa en la Ciudad de México. Debido a la popularidad del caso, Lafora se interesó en hacer un estudio clínico del personaje, al que diagnosticó con epilepsia psíquica, clasificación que contradecía al diagnóstico de esquizofrenia ofrecido por los médicos del Manicomio (donde Gregorio fue asilado en un principio). En una sesión de la Sociedad Mexicana de 
Neurología y Psiquiatría, en 1942, algunos médicos del Manicomio atacaron duramente los argumentos de Lafora y defendieron su propio diagnóstico (Ríos Molina, 2010, p.63-72; Huertas, 2002, p.53-56). Dicho conflicto alejó a Lafora de la comunidad neuropsiquiátrica hasta su partida a España en 1947.

Otro ejemplo es el de Dionisio Nieto. Para él existían dos formas de observar la neuropsiquiatría: la que correspondía a la corriente psicologista (enlazada al psicoanálisis y la psicobiología), representada en México por Erich Fromm, y que alegaba por la terapia psicológica; Nieto estaba en contra de esta forma de observar la enfermedad mental. Por otro lado, estaba la escuela organicista, a la que el español se adscribía, que afirmaba que toda enfermedad mental tenía un fundamento orgánico y que por lo tanto la utilización de fármacos y la investigación neurológica eran necesarias. Esta posición llevó a Nieto a remar contracorriente al grupo de médicos mexicanos que estaban del lado de la escuela psicologista y con los que debatió a lo largo de varios años. Sacristán (2007, p.337-338) menciona que Nieto sufrió un "doble exilio" en México ya que, además de su destierro, su convicción científica hizo que trabajara aislado durante mucho tiempo.

Un caso más fue el del Laboratorio de Estudios Médicos y Biológicos. Jaime Pi Suñer gestionó con la Fundación Rockefeller un financiamiento para abrir un centro de investigaciones científicas (donde estaría incluido lo concerniente al sistema nervioso) en México, donde pudieran laborar los españoles. Ese proyecto también fue apoyado económicamente por la Casa de España en México (institución creada por el gobierno de Lázaro Cárdenas para ayudar en lo económico y en lo académico a los exiliados) y por la Universidad Nacional; de hecho esta última designó un espacio dentro de las instalaciones de la Escuela Nacional de Medicina para establecer el Laboratorio. Así, cuando se creó en 1940, no fue un español quien estuvo a cargo, sino que por estar bajo la administración de la Universidad, un mexicano fue designado como su director: Ignacio González Guzmán (Exilio español, 2000, p.169, 188, 192). Por ello, ahí también trabajaron nativos como Efrén del Pozo o Clemente Villaseñor.

Pi Suñer y otros tuvieron que negociar sobre la pertinencia de crear un centro de ese tipo; sin embargo, su labor se vio favorecida porque justo en esos años la Universidad tuvo un crecimiento importante en el ámbito de la investigación científica. Además, la Casa de España, subsidiada por el gobierno federal de México, desempeñó un papel indispensable, ya que fungió como una intermediaria entre los españoles y las instituciones mexicanas, facilitó la inclusión laboral de los exiliados, y apoyó económicamente sus proyectos. Lo que se quiere poner de manifiesto es que sin ese contexto histórico los acogidos difícilmente hubieran podido incorporarse a la vida científica del país, por lo que tuvieron que buscar, negociar y/o crear espacios para ellos.

Entonces, el conocimiento sobre neurología de los médicos españoles fue importante en el sentido de que toda su preparación fue puesta en práctica en el terreno laboral en las instituciones médicas y de investigación, pero no se puede afirmar que solo con su arribo se definiera una nueva forma de practicar cualquier disciplina ligada a ella; por ejemplo, en el caso de la neurocirugía, la "del exilio dejó pocas huellas en México ... debido a la corta trayectoria de sus dos principales representantes", es decir, López Albo y Obrador (Dosil Mancilla, 2015, p.187). Y es que, además, no todos esos médicos se quedaron a vivir 
en México, ya que, en opinión de Dosil, algunos "españoles contemplaban el exilio como algo provisional" (Dosil Mancilla, 2010, p.373) por lo que hay que ver su participación como algo que fue parte de una integración, en donde los médicos nativos instruidos en EEUU también se desempeñaron. Resulta difícil pensar que los exiliados hubieren podido construir una escuela neurocientífica sin la intervención de los mexicanos, quienes más allá de ser considerados como "herederos lejanos de la escuela histológica de Cajal" (Dosil Mancilla, 2007, p.320), también participaron con la incorporaron otros saberes.

Los españoles llegaron a integrarse a una comunidad médica que ya estaba en formación, pues forjaron lazos profesionales con los mexicanos, ayudaron a la creación de centros de investigación e instituciones hospitalarias (como el Hospital Español), y fueron importantes docentes e investigadores en sitios como la Universidad Nacional y el Instituto Politécnico Nacional; no obstante, para que ello ocurriera fue necesaria la previa existencia de condiciones políticas y científicas adecuadas, y de elementos materiales y epistémicos que ya se desarrollaban en el país; cuestión que el mismo Nieto admitió: "Contrariamente a lo que se podía pensar, el panorama de la ciencia en México no era desolador. Ya había en el campo de la medicina grupos de investigadores" (Martínez Palomo, 2010, p.140).

Y por otro lado, habría que pensar en el impacto que tuvieron los españoles dentro de la asistencia pública en materia de neurología al momento de su llegada; es decir, los mexicanos estaban más interesados en tratar a los pacientes que muchas veces sobrepoblaban los hospitales públicos. La mayoría de los médicos nativos fue apoyada por el Estado para que saliera a especializarse, y por lo tanto su retribución debía estar dirigida a la terapéutica de enfermos neurológicos, situación que los españoles no podían satisfacer de inmediato, sobre todo por su desconocimiento de las problemáticas asistenciales del país.

No obstante, las redes que conformaron fueron indispensables, y por supuesto al igual que los nativos, ayudaron a que una comunidad neurológica en construcción se cohesionara debido a sus relaciones profesionales y académicas. Pero para poder comprender cómo se dio esa integración, hay que observar cuál fue el papel de los mexicanos en esta historia.

\section{Mexicanos en EEUU}

Desde la inauguración del Manicomio General de México (1910), la neurología y la psiquiatría estuvieron profundamente ligadas en el país y fueron conocidas con el nombre de neuropsiquiatría. De hecho, la institución psiquiátrica atendió a un número considerable de pacientes neurológicos (además de los psiquiátricos) hasta los años 1930.

En la década de 1920 algunos médicos mexicanos salieron al extranjero, principalmente a Europa, para estudiar neuropsiquiatría; por ejemplo, Leopoldo Salazar Viniegra se especializó en España y obtuvo su título en 1925 (Ríos Molina, 2016, p.31); la doctora Mathilde Rodríguez Cabo fue becada por la Sociedad Alexander von Humboldt en 1929 para instruirse en psiquiatría en Alemania (Sosenski, Sosenski, 2010, p.10); Alfonso Millán se tituló en París, hizo una especialidad en medicina legal y volvió en 1929 a México. Ellos regresaron al país para emplearse como médicos de planta en el Manicomio.

Ellos no se interesaron por estudiar neurología o neurocirugía, a pesar de que esta última era una disciplina que evolucionaba rápidamente en esos mismos años sobre todo en EEUU. 
Ello pudo deberse a que el objetivo de los médicos era aprender la clínica psiquiátrica tan necesaria en un país con un gran manicomio pero sin psiquiatras especializados, ya que tal práctica no se profesionalizó hasta la década de 1920 (Ríos Molina, 2016, p.26-39). No obstante, en palabras del neurocirujano Manuel Velasco Suárez (1989, p.126), el despunte de la neurología y la neurocirugía alcanzaría una gran importancia a partir de todos esos primeros neuropsiquiatras. De esta forma en los años 1920 y posteriores comenzó a conformarse una comunidad en medicina mental que fue la base para la introducción de una posterior clínica neurológica.

No fue hasta 1935 cuando salió al extranjero el primer mexicano para estudiar una rama de lo neurológico, en este caso, la neurocirugía: Clemente Robles (1907-2001). Se graduó de médico en 1929 y en 1935 fue becado por la Beneficencia Pública para ir a aleccionarse en cirugía fuera del país. Pasó por diversas clínicas en Minnesota (donde conoció a Dandy), Baltimore (ahí se instruyó con Cushing) y Nueva York, en EEUU. Fue ahí donde se abocó por el estudio de la neurocirugía y sus procedimientos más modernos porque, según sus palabras, en los países que visitó no encontró grandes diferencias con las técnicas quirúrgicas realizadas en México, a excepción de la ya citada. Él mencionó: "En donde nos aventajaban los cirujanos extraordinariamente era en neurocirugía ... porque en México yo no había visto operaciones del sistema nervioso ... y me empecé a aficionar a eso" (Robles, 1994, p.105).

A su regreso a México, en 1937, ingresó como médico en el Hospital General donde le fue asignado el Pabellón 19, un servicio general para pacientes de toda la institución; ahí reservó algunas camas para un servicio no oficial de neurocirugía que quedó integrado entre diciembre de 1937 y enero de 1938 en donde "ensayaría" las técnicas que había aprendido en EEUU. En 1938 las autoridades del Hospital lo cambiaron del Pabellón 19 al 7. Este último era para cirugía general, pero comenzó a orientarlo hacia la neurocirugía y juntó un grupo de cirujanos como Teodoro Guzmán, Alfredo Lejarza, Manuel Carbajal y Ramón del Cueto, a los que aleccionó para que lo auxiliaran en los procedimientos neuroquirúrgicos. Así fue como en ese mismo año Robles y sus colaboradores pudieron presentar el primer caso de extirpación de un tumor cerebral que se realizó con éxito en México (Robles, 1994, p.138; Fuentes, 1939, p.234). Robles realizó más operaciones, y debido a su trabajo el Consejo Consultivo y Técnico del Hospital designó al Pabellón 7 como un "servicio oficial de neurocirugía" en 1938 (Robles, 1994, p.139). Así fue como nació el primer departamento de ese tipo en México, y desde donde comenzó la inserción de conocimientos llegados desde EEUU que tenían relación con la neurología.

Un obstáculo con el que se topó Robles fue la falta de pacientes neuroquirúrgicos, según él, porque las autoridades médicas consideraban a la neurocirugía como una especialidad de casos raros y que no había que operar porque no todos los casos eran exitosos: "Era difícil conseguir enfermos; los médicos del hospital no colaboraban mucho, y era labor de uno ir a buscar los enfermos y traerlos. De las cuarenta camas que tenía el servicio apenas diez tenían enfermos de neurocirugía" (Robles, 1994, p.141, 24 febrero 1938). Ese es un ejemplo de las dificultades que pasó para integrar sus saberes a la medicina mexicana.

El proceso de integración fue lento, pero con pasos firmes. A raíz de la creación de tal servicio diversos médicos jóvenes y practicantes que llegaron al Hospital comenzaron a 
interesarse por la neurocirugía y, en palabras de Robles (1994, p.142), "se formó lo que se llamó en el Hospital 'la escuela del 7'”, donde se formaron cirujanos con experiencia en neurocirugía. Al mismo tiempo, otros colegas comenzaron a implementar esa práctica, como Felipe Aceves Zubieta, José Rojo de la Vega y Mariano Vázquez (Fuentes, 1939, p.233).

Robles reunió un grupo de trabajo con personas que supieran sobre clínica neurológica con la finalidad de tener un servicio de consulta externa. Sus principales colaboradores fueron médicos del Manicomio General, ya que ellos conocían mucho mejor la clínica neuropsiquiátrica; reclutó a Leopoldo Salazar Viniegra, Edmundo Buentello, José Quevedo y Mario Fuentes (Fuentes, Vázquez, 1939, p.493); sin embargo, ellos eran psiquiatras, no neurólogos. Esa no era una especialidad que existiera en México aún; de hecho, Robles fue el primer neurocirujano que tuvo el Manicomio cuando se abrió el servicio respectivo en 1943 (Oficio del visitador..., 24 abril 1937). Entonces se puede observar que, aunque la neurocirugía se introdujo fuera del Manicomio, la clínica neurológica seguía muy ligada a él en ese momento. Esa situación cambió cuando una nueva generación de médicos, que se especializaron en EEUU, regresó y comenzó a abrir departamentos de neurocirugía y clínica neurológica en diversas instituciones hospitalarias.

Si bien Robles fue un antecedente importante en la introducción de la neurocirugía en México, él no salió del país para estudiarla en sí, más bien se interesó en ella en el curso de sus viajes y su experiencia la obtuvo gracias a las operaciones que realizó en el Hospital General. Fueron los médicos posteriores a Robles que salieron a EEUU quienes trajeron consigo los conocimientos que desarrollaron casi de inmediato en la práctica médica y eso fue lo que dio paso a que, en las décadas siguientes, se conformara una comunidad de especialistas y se acrecentaran los servicios asistenciales en esa rama. Se debe prestar atención a un hecho muy particular: durante la década de 1920, algunos médicos se especializaron en el extranjero en neuropsiquiatría y dirigieron su práctica sobre todo a la clínica en el Manicomio; pero en la década de 1940, los que se instruyeron en neurocirugía ya no se acercaron tanto a la psiquiatría y no regresaron a trabajar en la institución mental.

El hecho de que los médicos se interesaran por la neurocirugía fue, entre otras cosas, porque los procedimientos avanzaban muy de prisa en EEUU. Pero, además de ello, pudieron viajar a estudiar al vecino del norte debido a una serie de condiciones que hay que describir. Como consecuencia de la Segunda Guerra Mundial (1939-1945), Europa perdió gran parte del protagonismo que había ostentado en materia científica durante las décadas anteriores; por esa razón, en opinión de Hobsbawm (1998, p.516-517), hubo un desplazamiento hacia EEUU como el principal lugar de atracción de la ciencia. Así, los que buscaban estudiar alguna disciplina científica, entre ellas las médicas, se alejaron del llamado "viejo continente", mientras que el país norteamericano atrajo a la mayoría de los recursos humanos que comenzaron a ingresar a institutos de investigación y universidades para especializarse.

Ese proceso se vio favorecido porque desde EEUU se empezó a promover la inmigración de estudiantes por medio de apoyos económicos; en ello participaron diversas instituciones como la Fundación Rockefeller, la cual no solo financió y participó en campañas sanitarias en varios países latinoamericanos desde su creación en 1913, sino que también impulsó la instrucción de médicos y otorgó becas para ir a estudiar a EEUU (Birn, 2006; Cueto, 2015); 
otras fundaciones que intervinieron de forma similar fueron la Carnegie y la Guggenheim. Pero también dependencias gubernamentales mexicanas como la Universidad Nacional, la Secretaría de Salubridad y Asistencia (SSA) o el Instituto Mexicano del Seguro Social (IMSS) igualmente financiaron viajes académicos. Así, esas instituciones funcionaron como un soporte económico que influyó en el aumento de la población de jóvenes mexicanos que fueron a estudiar a EEUU.

Sumado a eso, debido al involucramiento de México en la Segunda Guerra Mundial a favor de los aliados (1942), al reforzamiento de un panamericanismo científico y al surgimiento de una nueva diplomacia académica, se crearon las condiciones para un acercamiento entre los científicos estadounidenses y mexicanos (Saldaña, 1994, p.197198). De esta forma, el intercambio en ciencia y tecnología entre ambos países creció de manera extraordinaria en la década de 1940 a través de circuitos académicos, de ferias internacionales y por medio del financiamiento de proyectos bilaterales promovidos por fundaciones, empresas privadas y gobiernos. Ese fue el contexto en el que algunos médicos mexicanos se hicieron neurocirujanos en EEUU.

Tres personajes son lo que se han ubicado aquí, no solo porque fueron de los primeros en salir al extranjero a estudiar específicamente neurocirugía, sino también porque su trabajo fue abundante y escribieron diversas publicaciones: Manuel Velasco Suárez (1914-2001), Juan Cárdenas y Cárdenas (1912-1997) y Hernando Guzmán West (?-1979).

Manuel Velasco Suárez fue una de las figuras más influyentes en el ámbito médico y político para el desarrollo de la neurología y la neurocirugía en México. Después de graduarse de médico cirujano en 1939 en la Universidad Nacional, fue a hacer estudios de posgrado en neurocirugía en la Universidad de Harvard y en la Universidad George Washington, en EEUU, entre 1941 y 1943. A su regreso a México fue profesor de cirugía y clínica neurológica en la Escuela Nacional de Medicina y jefe del servicio de neurocirugía del Hospital Juárez. Debido su preparación y a sus conexiones políticas ostentó importantes cargos públicos concernientes a su disciplina, como ser el responsable de la Dirección de Neurología, Psiquiatría e Higiene Mental de la SSA en 1959. Además fue el principal impulsor del Instituto Nacional de Neurología y Neurocirugía (1964) (García Aguilar, 2000, p.21-26).

Juan Cárdenas y Cárdenas se graduó de médico en 1937 y obtuvo una beca en 1943 por parte de la SSA y la Fundación Rockefeller para hacer estudios en neurocirugía en centros universitarios y hospitalarios de EEUU y Canadá. A su regreso a México desempeñó el cargo de neurocirujano en el Hospital General y en el Manicomio, además de ser el jefe de consulta de neurología (Datos biográficos..., 1950). También tomó el cargo de director del Manicomio por un breve tiempo a inicios de la década de 1950 (Oficio de la Secretaría, 1 abr. 1952). Fue profesor en la Escuela Nacional de Medicina y en 1960 abrió un curso piloto para la especialización en neurocirugía en el Hospital Juárez.

Hernando Guzmán West, hijo del literato Martín Luis Guzmán, se graduó como médico cirujano en 1941 y en 1944 la Clínica Mayo (donde también conoció a Dandy) le otorgó una beca para especializarse en neurocirugía. Regresó en 1948 y se integró como neurocirujano en el Hospital General; posteriormente fue nombrado neurocirujano del Sanatorio Psiquiátrico del IMSS. Fue profesor de clínica neurológica en la Escuela Nacional de Medicina y jefe de la División de Cirugía del Hospital 20 de Noviembre (Zalce, 1980, p.41). 
Si bien no fueron los únicos que salieron al extranjero para estudiar, sí se debe advertir que ellos tres realizaron un trabajo de difusión en conjunto muy importante sobre neurocirugía y neurología, y eso ayudó a que posteriormente formaran grupos de trabajo con colegas que a la larga también se especializaron fuera del país; además, se integraron casi de inmediato al cuerpo docente de la Escuela Nacional de Medicina de la Universidad Nacional y con ellos "se inició una nueva etapa de la enseñanza y práctica de la neurología, impulsada por la neurocirugía" (Velasco Suárez, 1989, p.128). Estas palabras son importantes ya que la neurocirugía no solo implicó el establecimiento de una terapéutica particular en México, sino que también fue la puerta de entrada para el avance de toda la neurología, entendida como una disciplina que abarcaba varios rubros en la clínica y la investigación científica. En otras palabras, la neurocirugía, con sus posibilidades científicas y terapéuticas, trajo consigo un necesario desarrollo de las neurociencias (Revuelta, 10 feb. 2017).

Así, desde los años 1940, se proyectó la apertura de salas de neurocirugía y de investigación y clínica neurológica en varios centros hospitalarios, y esas ramas de la ciencia médica fueron en crecimiento. Ese proceso se nutrió con el inicio de la eclosión de una comunidad médica, que aquí ha sido denominada como la primera generación de neurólogos mexicanos. Entre ellos están: Eutimio Calzado Buentello que se especializó en la década de 1940 en neurocirugía y neurología en las Universidades de Rochester y de Columbia (ambas en Nueva York) y en la Sorbona (Francia), y a su regreso a México trabajó como neurólogo y neurocirujano en el Manicomio y en otros hospitales (Curriculum Vitae, 1962); Manuel Sánchez Garibay, que se instruyó en EEUU y que estableció el servicio de neurocirugía en el Hospital Central Militar; Luis Sáenz Arroyo, que fue becado por los laboratorios Eli Lilly y el IMSS para hacer investigación neuroanatómica en Boston, EEUU, entre 1947 y 1948 (Carta del Director, 23 dic. 1946); Gregorio González Mariscal, que entre 1949 y 1952 se especializó en neurocirugía en EEUU y en Europa (Carta de candidatura, 24 mar. 1965); María Cristina García-Sancho, que recibió una beca del gobierno chileno para aprender neurocirugía en el Instituto de Neurocirugía e Investigaciones Cerebrales de aquel país en 1949 (Rodríguez de Romo, Castañeda López, 2010); y Ramón del Cueto, que cursó un posgrado en neurocirugía en la Universidad de Pensilvania.

Los médicos mexicanos adoptaron una forma de entender la disciplina y al regresar al país aplicaron sus conocimientos. Y por supuesto que ellos también pasaron por un proceso de integración, pero fue diferente al de los españoles: en primer lugar, en lo político, ellos fueron apoyados por el Estado para salir del país, y por ello se esperaba que regresaran con resultados, planes y propuestas para mejorar la asistencia pública, y se les brindaron las facilidades para que así fuera; en segundo lugar, en el ámbito de la comunidad médica, no pasaron por los debates y dificultades que los exiliados para integrar su práctica, porque de hecho se esperaba que lo hicieran, y al no haber una comunidad de neurólogos conformada aún, la vinculación de sus saberes fue menos ríspida.

Por ejemplo, no hubo un choque o enfrentamiento académico entre los médicos del Manicomio y los neurocirujanos recién llegados ya que los últimos se emplearon en otros espacios que no se relacionaban con la práctica manicomial, por ello pudieron establecer una forma de concebir su disciplina sin restricciones. Y de hecho, cabe mencionar, justo en el periodo cuando esos médicos regresaban al país, los psiquiatras del Manicomio comenzaron 
a insistir sobre la necesidad de prohibir el ingreso de enfermos neurológicos, debido a que la institución se encontraba sobrepoblada, y afirmaron que ella había sido construida para asistir solo a pacientes psiquiátricos (Acta..., 4 ene. 1945). Fue en ese contexto que la unión entre psiquiatría y neurología comenzó a romperse en México.

Aun así, no se puede negar que existió algún conflicto entre la misma comunidad de neurólogos que se estaba creando; por ejemplo, Raúl Hernández Peón, neurofisiólogo especializado en EEUU, tuvo un enfrentamiento con su antiguo maestro, Velasco Suárez, cuando este último era director de neurología. Debido a ello, Hernández Peón se separó de su cargo como jefe de la Unidad de Investigaciones Cerebrales de la SSA y desde ahí tuvieron un alejamiento académico y personal que fue bien conocido por la comunidad en su momento (Drucker Colín, Rojas, 26 oct. 2016; Revuelta Gutiérrez, 10 feb. 2017).

Ahora bien, el proceso de integración de saberes contuvo elementos que hay que detallar. Un factor principal que caracterizó a los neurólogos nativos fue que implantaron una estructura de práctica médica adquirida en EEUU, donde la neurocirugía y la neurología respondieron a necesidades bien definidas que tenían que ver con la práctica clínica y la terapéutica de enfermos, siempre unida a la investigación científico-médica. Esa tradición de enseñanza médica se originó a consecuencia de la redacción del libro Medical education in the United States and Canada (mejor conocido por el nombre de Flexner Report), publicado en 1910 por el médico Abraham Flexner. El texto tenía el objetivo de estandarizar la enseñanza superior de la medicina en las universidades estadounidenses y con esa obra se consiguió establecer lineamientos para la instrucción de médicos, clausurar centros educativos que no cumplieran con las normas, y se creó un modelo generalizado de enseñanza que se perfeccionó en la década de 1930. En esa nueva estructura era menester que los estudiantes llevaran a cabo trabajo clínico para la asistencia y la terapéutica de enfermos, al mismo tiempo que se les aleccionaba como investigadores (Flexner, 1910, p.53-55).

Es verdad que para mediados del siglo XX la influencia del Flexner Report había llegado a las escuelas de medicina de Europa y América, incluyendo a las de España y México. Sin embargo, hay que destacar dos cosas: la primera es que los médicos mexicanos estudiaron en el país donde surgieron las reformas propuestas por dicho informe, es decir, se impregnaron de esas ideas de primera mano; la segunda es que, cuando volvieron al país, con apoyo del Estado pusieron en práctica casi de inmediato ese modelo de enseñanza y clínica en las instituciones públicas mexicanas que se estaban conformando. En ese sentido, los nativos tenían cierta libertad para acoplar lo aprendido afuera; cosa que los españoles no tuvieron debido a su propio proceso de exilio, a pesar de haberse apropiado de elementos emanados del Flexner Report.

Los mexicanos asumían la necesaria unión entre terapéutica e investigación científica, pero a diferencia de los estadounidenses, y de los otrora españoles, tenían un factor agregado: la responsabilidad de satisfacer las necesidades asistenciales y terapéuticas del país. Dicho agregado estuvo directamente ligado con los intereses del Estado posrevolucionario dirigido, entre otras cosas, a satisfacer necesidades sociales y de salud con fines de gobernabilidad. Esa puede ser una razón por la que dichos médicos obtuvieron el apoyo de los gobiernos que financiaron los nuevos espacios para su práctica, como fueron: la sala de neurocirugía 
del Manicomio General (1943); el departamento de neurocirugía pediátrica en el Hospital Infantil de México; el servicio de neurología y neurocirugía del Hospital de La Raza (1955); en 1950 se creó en el Hospital Juárez un servicio de neurocirugía de la mano de Velasco Suárez; en 1958 se creó la Unidad de Neurología y Neurocirugía del Hospital General; en el Hospital 20 de Noviembre se abrió el servicio de neurocirugía correspondiente (1961); en 1962 se creó el departamento de neurología y neurocirugía del Centro Médico Nacional (Robles, 1994; Velasco Suárez, 1989); y en 1964 se fundó el Instituto Nacional de Neurología y Neurocirugía.

Toda esa nueva estructura fue coadyuvada por médicos mexicanos que aprovecharon las políticas públicas emanadas del Estado; esta situación la desconocían los exiliados a su llegada, pero gracias a sus saberes en neurología, neurocirugía y neurociencias se integraron a lo que se comenzaba a crear localmente. Esa es quizá la diferencia más importante entre los nativos y los exiliados: los primeros salieron a especializarse con el objetivo de volver para poner en práctica en instituciones hospitalarias sus conocimientos, y esa es acaso la razón por la que participaron en menor medida en la investigación neurocientífica "pura"; los segundos arribaron al país por condiciones políticas ajenas a ellos y por eso se vieron en la necesidad de integrarse a los espacios que pudieron encontrar y/o crear, y por supuesto una vez en el país de acogida también participaron en la satisfacción de las necesidades asistenciales, pero al mismo tiempo buscaron espacios para continuar con su labor de investigación científica que quedó truncada con la Guerra Civil y, por eso, quizá, estuvieron más cercanos a las neurociencias. En ese sentido, fue la comunidad conformada por mexicanos la que creó las reglas de integración, que ellos mismos tuvieron que seguir, pero a las que los españoles tuvieron que adecuarse.

\section{Consideraciones finales}

El surgimiento y desarrollo de la neurología en México respondió tanto a condiciones externas como internas. La presencia de los médicos españoles fue fundamental, ya que tenían importantes conocimientos, tanto por ser herederos del trabajo de Cajal, como por haberse instruido en otros espacios europeos. Sin embargo, ellos no conocían las instituciones, las redes académicas y mucho menos las necesidades del país; así, tuvieron que llegar a integrarse a un terreno desconocido, buscando espacios donde insertarse y creando otros para llevar a cabo su práctica. Entonces, gracias a las instituciones que ayudaron a fundar y a las que llegaron a laborar, a la docencia que impartieron, y a las relaciones profesionales que establecieron, pasaron por una integración a veces ríspida, otras cooperativa, pero al final fructífera. En opinión de Martínez Palomo (2010, p.131), "los médicos exiliados españoles llegaron a México en el momento más oportuno", ya que en el país existían las condiciones idóneas para continuar su labor; no obstante, eso implica que la neurología y las neurociencias no pudieron despuntar solo por su presencia. Por ello fue preciso revisar lo que hicieron los nativos al respecto.

Los médicos mexicanos trajeron un bagaje de saberes que pusieron en práctica en instituciones hospitalarias públicas. Al mismo tiempo, ellos comenzaron a formar redes, tanto institucionales como docentes que permitieron que en pocos años eclosionara una 
comunidad médica, con tendencias a satisfacer las necesidades asistenciales del país, mediante la clínica, la terapéutica y la investigación científica. Su integración tuvo que ver más con la creación de espacios y la introducción de conocimientos que eran casi inexistentes en el país; en otras palabras, salieron con el objetivo de regresar e integrar lo aprendido. Ellos no entraron en conflicto con otros médicos, ni siquiera con los psiquiatras que eran, acaso, los médicos más cercanos a su práctica. Y los enfrentamientos que hubo se dieron al interior de la comunidad que habían ayudado a crear, una vez que ellos mismos establecieron las reglas de integración.

Al final, ambos grupos fueron parte de un mismo proceso de acumulación de conocimientos que creció exponencialmente y que a la larga llevó a la profesionalización de la práctica, a la conformación de una especialización impartida oficialmente en el país, y a la institucionalización de la neurología en México. Todo ello resultado del trabajo realizado entre exiliados y nativos.

\section{REFERENCIAS}

ACTA de la $31^{\text {a }}$ Junta del Consejo Psiquiátrico de Toxicomanías e Higiene Mental del Manicomio General. Fondo Manicomio General, Sección Administrativa, caja 48, expediente 2, f.47-50 (Archivo Histórico de la Secretaría de Salud, Ciudad de México). 4 ene. 1945.

BARATAS DÍAZ, Alfredo. La investigación biológica en la Junta a través de la figura de Pío del Río Hortega: una parábola sobre el desarrollo científico español del primer tercio del siglo XX. In: Sánchez Ron, José Manuel et al. El laboratorio de España: La Junta para Ampliación de Estudios $y$ de Investigaciones Científicas. Madrid: Consejo Superior de Investigaciones Científicas, 2007. p.333-355.

BIRN, Anne-Emanuelle. Marriage of convenience: Rockefeller international health and revolutionary Mexico. Rochester, NY: University of Rochester Press. 2006.

CARTA DE CANDIDATURA de Gregorio González Mariscal para ingresar a la Academia Nacional de Medicina. Fondo Facultad de Medicina, Sección Personal Académico, volumen 44, expediente 717, f.26 (Archivo Histórico de la Faculta de Medicina de la Universidad Nacional Autónoma de México, Ciudad de México), 24 mar. 1965.

CARTA DEL DIRECTOR de la Escuela Nacional de Medicina al Dr. Mario Quiñones. Fondo Facultad de Medicina, Sección Personal Académico, volumen 117, expediente 2145, f.19 (Archivo Histórico de la Faculta de Medicina de la Universidad Nacional Autónoma de México, Ciudad de México). 23 dic. 1946.
CRUZ-SÁNCHEZ, Félix et al. La escuela española de neurología cajaliana. La semilla iberoamericana. Revista Española de Patología, v.35, n.4, p.487-492, 2002.

CUETO, Marcos. An asymmetrical network: national and international dimensions of the development of mexican physiology. Journal of the History of Medicine and Allied Sciences, v.71, n.1, p.43-63, 2015.

CURRICULUM VITAE. Eutimio Calzado Buentello. Fondo Facultad de Medicina, Sección Personal Académico, volumen 16, expediente 250, f.7-8 (Archivo Histórico de la Facultad de Medicina de la Universidad Nacional Autónoma de México, Ciudad de México), 1962.

DATOS BIOGRÁFICOS del Dr. Juan Cárdenas y Cárdenas. Gaceta Médica de México, t.80, n.3-4, p.238, 1950.

DÍAZ, J.L. El legado de Cajal en México. Revista de Neurología, v.48, n.4, p.1-9, 2009.

DOSIL MANCILLA, Francisco Javier. La escuela de Cajal en México: diplomacia oficiosa, representación y redes. In: Herrera León, Fabián. Diplomacia oficiosa, representaciones y redes extraoficiales en la historia de América Latina. Ciudad de México: Universidad Nacional Autónoma de México, 2015. p.167-197.

DOSIL MANCILLA, Francisco Javier. ¡A la salud de México! Médicos españoles exiliados para la sanidad de un país en transformación. In: Dosil Mancilla, Francisco Javier; Sánchez Díaz, Gerardo. Continuidades y rupturas: una historia tensa de la ciencia en México. Ciudad de México: Universidad Michoacana de San Nicolás de 
Hidalgo; Universidad Nacional Autónoma de México, 2010. p.363-392.

DOSIL MANCILLA, Francisco Javier. La estela de Cajal en México. Arbor: Ciencia, Pensamiento y Cultura, v.185, n.735, p.29-40, 2009.

DOSIL MANCILLA, Francisco Javier. La JAE peregrina. Revista de Indias, v.67, n.239, p.307332, 2007.

DRUCKER COLÍN, René; ROJAS, José Antonio. Raúl Hernández Peón. Disponible en: http:// www.uam.mx/e_libros/biografias/HERNANDEZPEON.pdf. Acceso en: 26 oct. 2016.

EXILIO ESPAÑOL y ciencia mexicana: génesis del Instituto de Química y del Laboratorio de Estudios Médicos y Biológicos de la Universidad Nacional Autónoma de México (1939-1945). Ciudad de México: El Colegio de México; Universidad Nacional Autónoma de México, 2000.

FERNÁNDEZ GUARDIOLA, Augusto. Las neurociencias en el exilio español en México. Ciudad de México: Fondo de Cultura Económica, 1997.

FLEXNER, Abraham. Medical education in the United States and Canada. Stanford, CA: Carnegie Foundation, 1910.

FUENTES, Mario. Estado actual de la cirugías de los tumores del cerebro en nuestro medio y consideraciones diagnósticas. Archivos de Neurología y Psiquiatría de México, t.2, n.5, p.231248, 1939.

FUENTES, Mario; VÁZQUEZ, Mariano. Un caso de tumor del tercer ventrículo. Archivos de Neurología y Psiquiatría de México, t.3, n.2, p.493508, 1939.

GARCÍA AGUILAR, María del Carmen. Manuel Velasco Suárez: del gabinete científico al despacho político. Tuxtla Gutiérrez: Consejo Estatal para la Cultura y las Artes de Chiapas, 2000.

GIRAL, Francisco. Ciencia española en el exilio (1939-1989): el exilio de los científicos españoles. Barcelona: Anthropos; Madrid: Centro de Investigación de Estudios Republicanos, 1994.

GUERRA, Francisco. La medicina en el exilio republicano. Madrid: Universidad de Alcalá, 2003.

GUERRA, Francisco. Médicos españoles en el exilio. Bilbao: Fundación Ramón Rubial, 1996.

HOBSBAWM, Eric. Historia del siglo XX. Buenos Aires: Grijalbo, 1998.

HODELÍN TABLADA, Ricardo. Contribución de las controversias entre Cushing y Dandy al desarrollo de la neurocirugía. Revista Médica Electrónica, v.33, n.7, p.835-844, 2011.
HUERTAS, Rafael. Los médicos de la mente: de la neurología al psicoanálisis. Madrid: Nivola, 2002.

LAÍN ENTRALGO, Pedro. Cajal por sus cuatro costados. In: Ramón y Cajal, 1852-1934. Madrid: Ministerio de Educación y Ciencia, 1978. p.17-65.

LIDA, Clara E. Inmigración y exilio: reflexiones sobre el caso español. Ciudad de México: El Colegio de México; Siglo XXI Editores, 1997.

MARTÍNEZ PALOMO, Adolfo. Médicos de La Casa de España. In: Los refugiados españoles y la cultura mexicana. Ciudad de México: El Colegio de México, 2010. p.129-143.

NIETO, Dionisio. La influencia de Cajal en América. In: Nieto, Adela. La obra científica de Dionisio Nieto. Ciudad de México: Universidad Nacional Autónoma de México, 1990. p.19-24.

OFICIO DE LA SECRETARÍA de Salubridad y Asistencia al director del Manicomio General. Fondo Manicomio General, Sección Administrativa, caja 17, expediente 6, f.24 (Archivo Histórico de la Secretaría de Salud, Ciudad de México), 1 abr. 1952.

OFICIO DEL VISITADOR General de la Beneficencia Pública al director del Manicomio General. Fondo Manicomio General, Sección Administrativa, caja 30, expediente 63, f.68 (Archivo Histórico de la Secretaría de Salud, Ciudad de México), 24 abril 1937.

REVUELTA GUTIÉRREZ, Rogelio. [Entrevista]. Entrevistador: Daniel Vicencio. Ciudad de México. Documento sonoro digital (1h10min). Entrevista concedida para tesis doctoral en historia. 10 feb. 2017.

RÍOS MOLINA, Andrés. Cómo prevenir la locura. Psiquiatría e higiene mental en México, 19341950. Ciudad de México: Universidad Nacional Autónoma de México; Siglo XXI, 2016.

RÍOS MOLINA, Andrés. Memorias de un loco anormal: el caso de Goyo Cárdenas. Ciudad de México: Debate, 2010.

ROBLES, Clemente. Evocaciones, 1920-1980. Ciudad de México: Instituto Mexicano del Seguro Social; Academia Nacional de Medicina; Archivo de la Palabra Médica, 1994.

ROBLES, Clemente. Carta al director del Manicomio General. Fondo Manicomio General, Sección Administrativa, caja 36, expediente 3, f.1 (Archivo Histórico de la Secretaría de Salud, Ciudad de México). 24 feb. 1938.

RODRÍGUEZ DE ROMO, Ana Cecilia; CASTAÑEDA LÓPEZ, Gabriela. María Cristina García-Sancho y Álvarez Tostado: primera neurocirujana en Latinoamérica. Salud Mental, v.33, n.2, p.111-121, 2010. 
RODRÍGUEZ QUIROGA, Alfredo. Sobre las investigaciones neurofisiológicas de la escuela histológica cajaliana: la correspondencia entre Santiago Ramón y Cajal y Rafael Lorente de Nó (1930-1934). Dynamis: Acta Hispánica ad Medicinae Scientiarumque Historiam Illustrandam, v.22, p.411-435, 2002.

SACRISTÁN, Cristina. En defensa de un paradigma científico: el doble exilio de Dionisio Nieto en México, 1940-1985. In: Campos Marín, Ricardo et al. De la "Edad de Plata" al exilio: construcción y "reconstrucción" de la psiquiatría española. Madrid: Frenia, 2007. p.327-346.

SALDAÑA, Juan José. El sector externo y la ciencia nacional: el conservacionismo en México (1934-1952). Quipu: Revista Latinoamericana de Historia de la Ciencia, v.11, n.2, p.195-217, 1994.

SHERMAN, Irving J. et al. Personal collections of Walter E. Dandy and his Brain Team. Journal of Neurosurgery, v.105, n.3, p.487-493, 2006.
SOSENSKI, Susana; SOSENSKI, Gregorio. En defensa de los niños y las mujeres: un acercamiento a la vida de la psiquiatra Mathilde Rodríguez Cabo. Salud Mental, v.33, n.1, p.1-10, 2010.

VELASCO SUÁREZ, Manuel. Neurocirugía. In: Soberón, Guillermo et al. La salud en México: testimonios 1988, t.4, v.2. Ciudad de México: Secretaría de Salud; Fondo de Cultura Económica, 1989. p.123-151.

VÉLEZ CARDONA, Waldemiro. La integración del conocimiento como fundamento de los estudios generales. Ciencia y Sociedad, v.38, n.4, p.643-657, 2013.

WEISZ, George. Divide and conquer: a comparative history of medical specialization. Oxford: Oxford University Press, 2005.

ZALCE, Horacio. Hernando Guzmán West. In memoriam. Gaceta Médica de México, t.117, n.1, p.41-42, 1980. 